\title{
LA FIJACIÓN EXTERNA EN CIRUGÍA RECONSTRUCTIVA: COLGAJO DE PIERNAS CRUZADAS.
}

\section{EXTERNAL FIXATION IN RECONSTRUCTIVE SURGERY: CROSSED LEG FLAP.}

\author{
Andrés Aguilar Ezquerra ${ }^{1,3}$, Valentín Yuste Benavente ${ }^{1}$, Elena Jordán Palomar².
}

\begin{abstract}
Resumen:
Introducción: Los defectos de cobertura del miembro inferior van asociados en ocasiones con la disrupción de los pedículos vasculares que imposibilitan la realización de colgajos libres, siendo en estos casos el colgajo de piernas cruzadas una buena alternativa terapéutica. Material y métodos: se presentan 2 casos de pacientes que tras ser intervenidos en múltiples ocasiones en los miembros inferiores, presentaban un defecto de cobertura no tratable con un colgajo libre microvascularizado. Se realizó un colgajo de piernas cruzadas estabilizando ambas extremidades inferiores con un fijador externo. Ambos pacientes mostraron una recuperación satisfactoria con una vuelta a la deambulación sin ayudas adicionales. Conclusión: El colgajo de piernas cruzadas estabilizado mediante fijación externa es una alternativa válida para defectos de partes blandas.
\end{abstract}

Palabras clave: colgajo de piernas cruzadas; fijador externo..

\section{Abstract:}

Introduction: Lower limb coverage defects are sometimes associated with disruption of vascular pedicles that make it impossible to perform free flaps. In these cases the crossed leg flap is a good therapeutic alternative. Material and methods: Two patients presented a coverage defect non-treatable with microvascularized free flap after being operated on multiple times in the lower limbs. A cross leg flap was performed stabilizing both lower limbs with an external fixator. Both patients showed a satisfactory recovery returning to walk without additional help. Conclusion: Crossed leg flap stabilized by external fixation is a valid alternative for soft tissue defects.

Keywords: crossed leg flap; external fixator.

\footnotetext{
1 Doctor en Medicina. Hospital Universitario Miguel Servet, Zaragoza, España.

2 Médico especialista en Cirugía Plástica y Reparadora. Hospital Universitario Miguel Servet, Zaragoza, España.

3 Email de contacto: aaguilarez@gmail.com
} 


\section{Introducción}

Actualmente muchos casos de traumatismos, accidentes de tráfico, electrocuciones o úlceras por problemas venosos o arteriales llevan asociado a corto o medio plazo defectos de partes blandas en la pierna, que obligan a la colaboración de los servicios de cirugía plástica y traumatología, ya sea para el tratamiento del problema traumático como del defecto de cobertura de partes blandas.

Muchas veces estas graves lesiones requieren de la realización de colgajos libres o microvascularizados, que en ocasiones no se pueden realizar debido al daño de los troncos arteriales ya sea por el traumatismo inicial como por las sucesivas intervenciones que requieren estos casos.

En estas ocasiones, los colgajos de piernas cruzadas representan una buena opción terapéutica, ya que a pesar de los impedimentos para la movilización del paciente y la dificultad para que se sienta cómodo con las piernas en la misma posición durante 3 ó 4 semanas (tiempo necesario para la aparición de neovasos), han demostrado unos resultados satisfactorios en defectos de miembros inferiores con exposición de material de osteosíntesis, hueso o tendón ${ }^{(1)}$.

\section{Material y Método}

Presentamos 2 casos derivados de otros hospitales a nuestro centro, que fueron tratados por el servicio de Cirugía plástica y Reparadora en colaboración con el servicio de Traumatología.

\section{Caso 1}

Se trata de un varón intervenido en 13 ocasiones en la extremidad inferior izquierda por unos pies cavos congénitos, que finalizó su tratamiento con un clavo de artrodesis retrógrado tibio-talo-calcáneo. El paciente presentaba un defecto de partes blandas sobre la zona posterior del maléolo externo (figura 1).

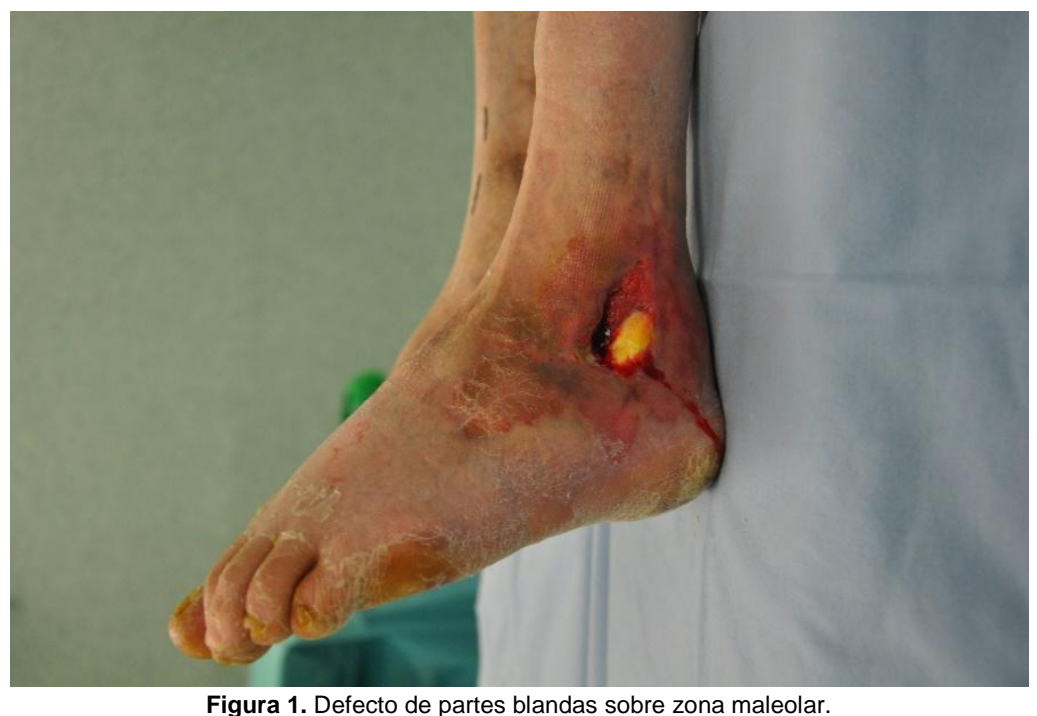

Tras el estudio angiográfico se objetivó que no había arterias disponibles para realizar un colgajo libre, por lo que se planteó un colgajo de piernas cruzadas.

Para ello se realizó una intervención quirúrgica conjunta en que mientras el cirujano plástico diseñaba y preparaba el colgajo el traumatólogo colocaba los pines del fijador esquivando el material de la osteosíntesis previa; siempre teniendo en cuenta el sitio en que la extremidad derecha iba a quedar situada. Tras este paso se procedió a la sutura del colgajo y fijación externa de ambas extremidades inferiores, siendo este paso indispensable para conseguir una estabilidad suficiente para el prendimiento del colgajo (figura 2). 


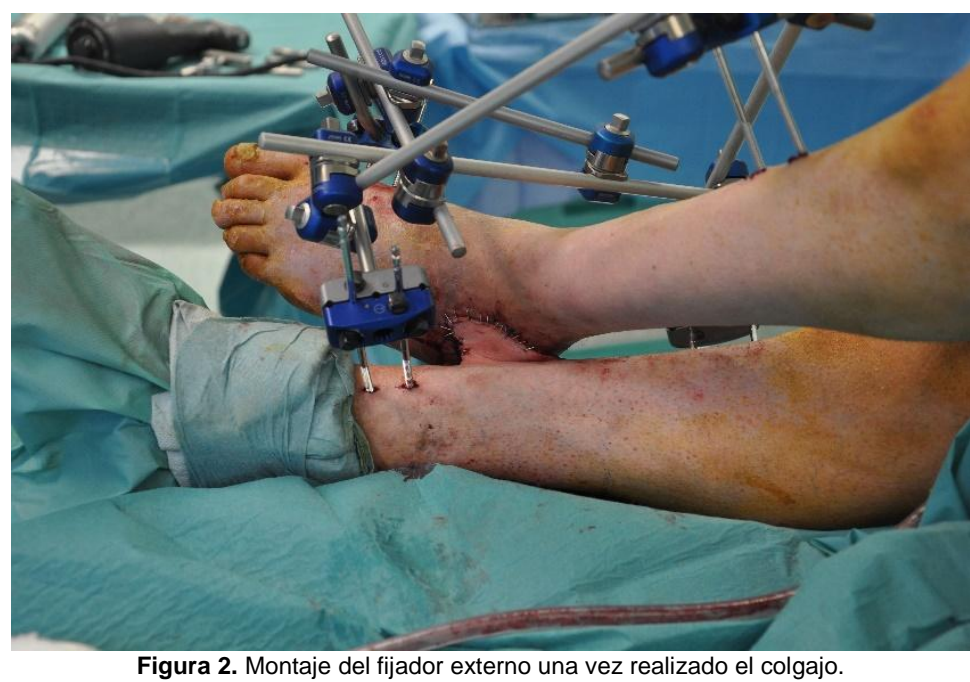

Al paciente se le colocó un catéter de anestesia epidural, y el fijador externo fue mantenido durante 3 semanas, cuando se procedió a la retirada del mismo y sección del pedículo del injerto (figura 3).

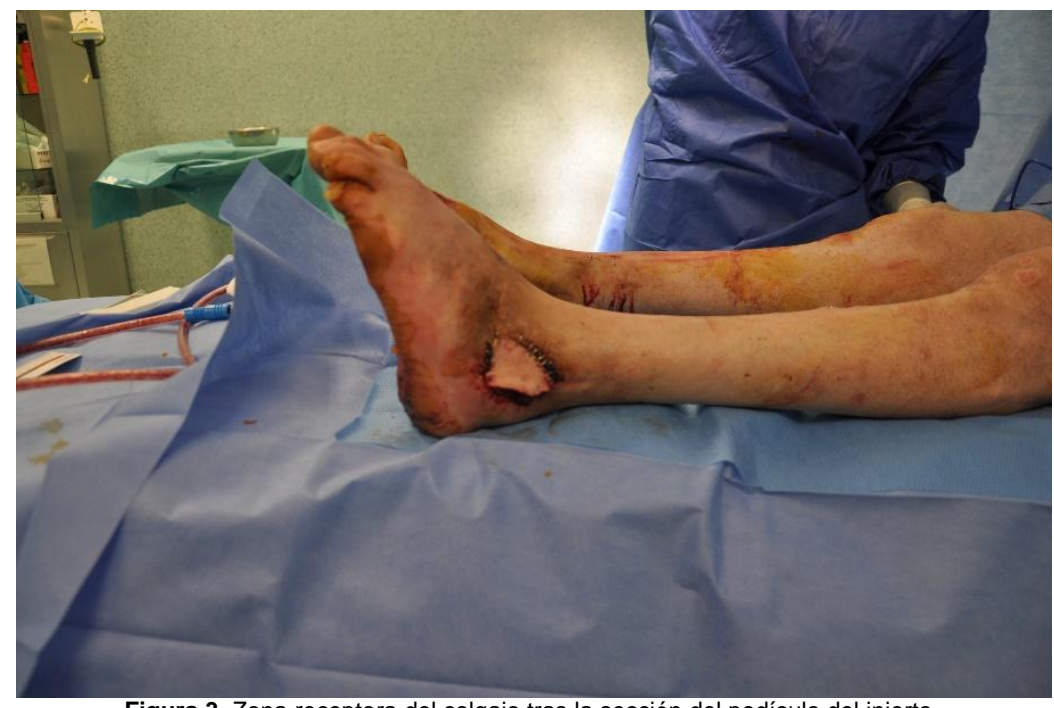

Figura 3. Zona receptora del colgajo tras la sección del pedículo del injerto.

El paciente volvió a deambular con normalidad, usando dos muletas que fueron retirándose progresivamente, y no necesitó una rehabilitación específica.

\section{Caso 2}

El segundo caso se trata de una paciente de 40 años, intervenida múltiples veces en otro centro tras sufrir fractura de ambas tibias y peronés. A su llegada a nuestro centro, presentaba un defecto en zona anterior de tibia derecha con exposición de material de osteosíntesis, que fijaba un injerto osteomuscular de escápula que fracasó (figura 4). 


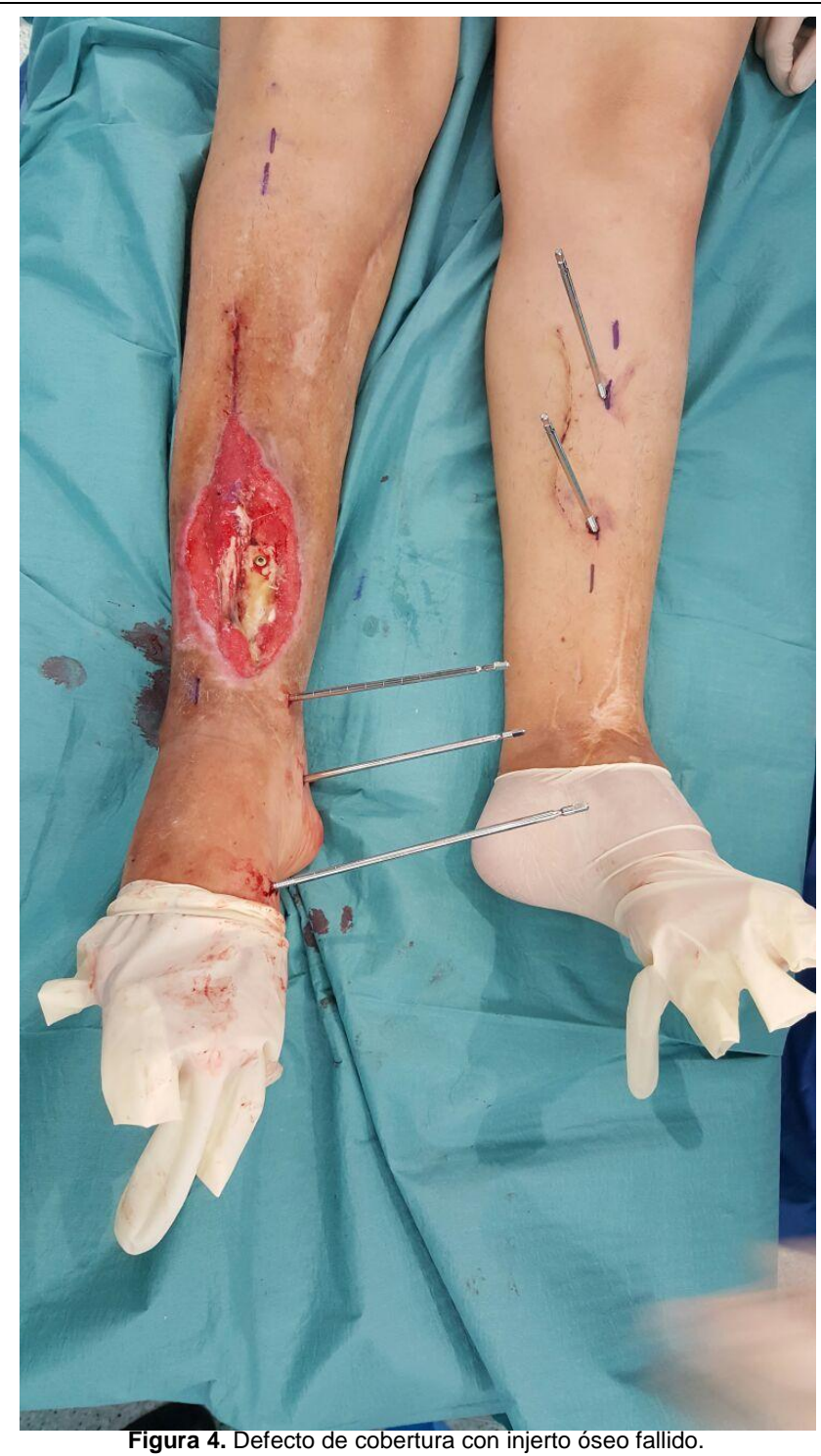

Debido a las múltiples intervenciones llevadas a cabo, la única solución planteada por el equipo de cirugía plástica fue el de realizar un colgajo de piernas cruzadas.

Esta intervención se llevó a cabo de manera similar a lo anteriormente expuesto tras la retirada del tornillo y el injerto óseo no viable, con la colaboración conjunta y simultánea de cirujanos plásticos y traumatólogos, se realizó la colocación de fichas y el colgajo a la vez (figura 5), y tras la sutura del colgajo sobre su lecho se finalizó la fijación externa (figura 6), que se mantendría durante 3 semanas hasta la sección del pedículo del injerto. En este caso también se usó catéter epidural, y la paciente volvió a deambular sin necesidad de una rehabilitación específica, tras un periodo de deambulación asistida con muletas.

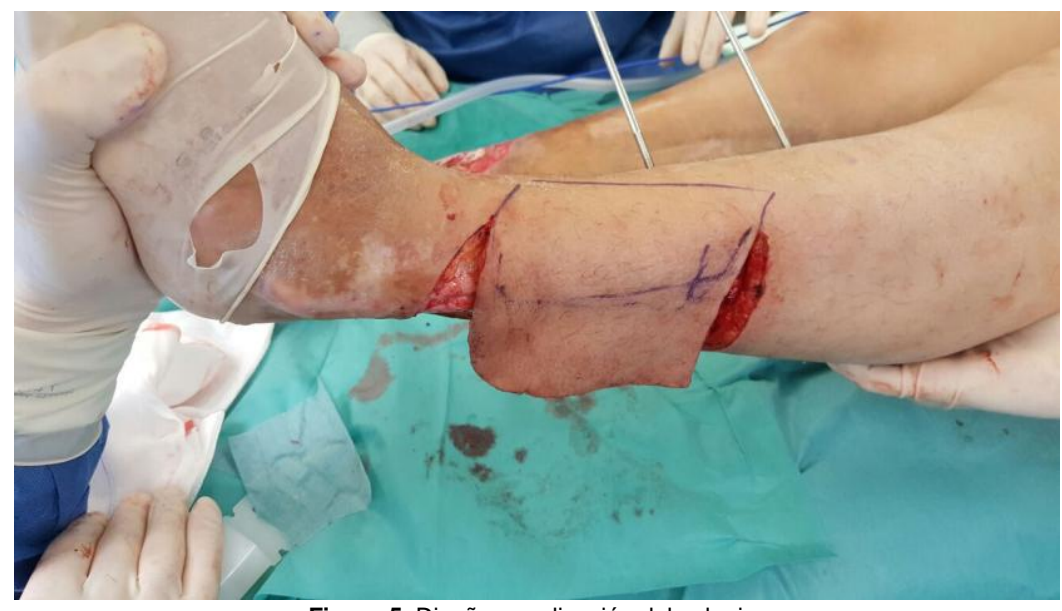

Figura 5. Diseño y realización del colgajo. 


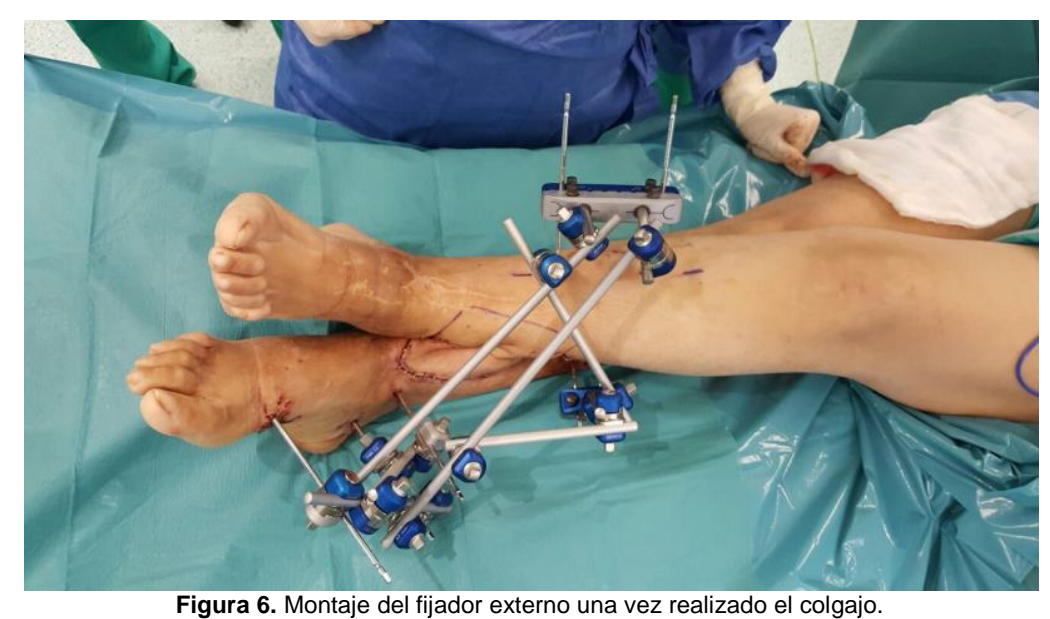

\section{Discusión}

Los colgajos de piernas cruzadas han sido ampliamente usados desde hace muchos años para el tratamiento de defectos de cobertura en miembros inferiores, cualquiera que sea su causa. El principal problema de esta técnica es la inmovilización de ambas extremidades inferiores hasta el prendimiento del injerto, por lo que en los últimos años se ha optado más por la realización de colgajos pediculados.

Sin embargo, en casos en que por el traumatismo sufrido por la extremidad inferior no se dispone de vasos para realizar una anastomosis satisfactoria, el colgajo de piernas cruzadas es una alternativa que ha demostrado buenos resultados.

El colgajo de piernas cruzadas ha sido utilizado con éxito en defectos de cobertura en zona pretibial ${ }^{(2)}$, zona en que debido a la precaria vascularización cutánea es frecuente encontrar fracaso de partes blandas.

Esta técnica ha demostrado buenos resultados en el tratamiento de defectos de cobertura asociados a traumatismos graves, como fracturas de pilón tibial de alta energía ${ }^{(3)} \mathrm{o}$ tras sufrir accidentes de tráfico ${ }^{(4)}$, definiéndose por algunos autores como la alternativa preferida a los colgajos libres en el tratamiento de defectos de partes blandas postraumáticos del miembro inferior ${ }^{(5,6)}$. Algunos autores incluso han usado el colgajo de piernas cruzadas asociado a terapia VAC de presión negativa con resultados satisfactorios ${ }^{(7)}$.

También ha sido usada con éxito en quemaduras de alto voltaje de segundo y tercer grado que afectan a miembro inferior y pie cuando no se pueden realizar colgajos libres debido a los daños en la extremidad afectada $^{(8)}$.

Algunos autores publican resultados satisfactorios en defectos en extremidades inferiores en niños, siendo una buena alternativa al tratamiento debido a la dificultad de realizar colgajos microvascularizados en pacientes pediátricos por el pequeño calibre de los vasos y por las lesiones de los mismos tras haber sufrido traumatismos importantes ${ }^{(9)}$.

También esta técnica se ha usado para realizar colgajos neurofasciocutáneos en lesiones en pies con buen resultado estético y funcional a medio-largo plazo ${ }^{(10)}$, siendo satisfactorio tanto respecto a la deambulación como a la recuperación de la sensibilidad cutánea.

\section{Conclusiones}

Podemos concluir que el colgajo de piernas cruzadas es un importante recurso para el cirujano que puede usarse para resolver situaciones complejas, como las mencionadas en los casos aquí expuestos.

Asimismo, para su correcta realización es necesario un profundo conocimiento de la fijación externa, siendo en muchas ocasiones necesaria la colaboración con el traumatólogo para la correcta colocación del fijador que asegure una adecuada estabilidad, acortando el tiempo quirúrgico al trabajar ambos profesionales a la vez. 


\section{Bibliografía}

1.- Reisler T1, Buziashvili D2, Liu FC2, Datiashvili RO1. Revisiting the Fasciocutaneous Perforator Cross-Leg Flap. Eplasty. 2016 Apr 28;16:ic16. eCollection 2016.

2.- Melikoglu C1, Eren F, Kok D. Repair of pretibial atonic wound with long cross-leg flap. Int J Low Extrem Wounds. 2013 Sep;12(3):223-5.

3.- Sood A1, Khamsi B, Datiashvili R, Berberian WS. Use of cross-leg flap for wound complications resulting from open pilon fracture. Am J Orthop (Belle Mead NJ). 2015 Mar;44(3):131-4.

4.- Macedo JLS1,2, Rosa SC1,3, Botelho DL1, Santos CPD1, Queiroz MN2, Gomes TGACB2. Lower extremity reconstruction: epidemiology, management and outcomes of patients of the Federal District North Wing Regional Hospital. Rev Col Bras Cir. 2017 Jan-Feb;44(1):9-16.

5.- Halls MJ1. Pedicled cross-leg flaps. J Am Coll Surg. 2014 Mar;218(3):503-4.

6.- Momeni A1, Buntic RF2, Buncke GM2. Cross-leg flaps: preferred alternative to free flaps? J Am Coll Surg. 2014 Feb;218(2):308-9.

7.- Pan X1, Wang G2, Lui TH3. Transplantation Treatment of Extensive Soft-Tissue Defects in Lower Extremities with a Combination of Cross-Bridge Flap and Combined Free-Tissue Flap Covered by Vacuum Sealing Drainage: One Case Report. Open Orthop J. 2017 Jul 31;11:704-713.

8.- Eser C1, Kesiktaş E2, Gencel E2, Aslaner EE2, Yavuz M2. An alternative method to free flap for distal leg and foot defects due to electrical burn injury: distally based cross-leg sural flap. Ulus Travma Acil Cerrahi Derg. 2016 Jan;22(1):46-51.

9.- Kapadia A1, Cheruvu VR, John JR, Tripathy S, Sharma RK. Reconstruction in pediatric orthopedic trauma: the role of primary cross leg flap cover. J Pediatr Orthop B. 2017 Mar;26(2):172-178.

10.- Wang ZQ1, Cao YL2, Huang YF2, Liu DQ2, Li XF2. Cross-leg repair of large soft-tissue defects in distal sites of the feet by distally based neuro-fasciocutaneous flaps with perforating vessels. Genet Mol Res. 2014 Jul 25;13(3):5484-91. 